\title{
Fatores associados à limitação de atividade em casos novos de hanseníase em município hiperendêmico do Nordeste, Brasil: estudo transversal
}

\author{
Factors associated with activity limitation in new leprosy cases in a Northeastern hyperendemic \\ municipality, Brazil: a cross-sectional study
}

\section{Factores asociados con la limitación de la actividad en nuevos casos de lepra en un municipio hiperendémico del noreste, Brasil: un estudio transversal}

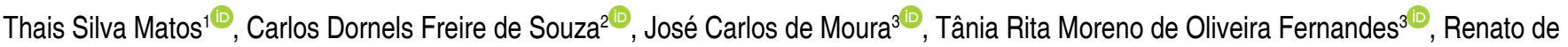 \\ Souza Mariano4 ${ }^{(\mathbb{D}}$ \\ ${ }^{1}$ Faculdade São Francisco de Juazeiro - FASJ, Juazeiro, Bahia, Brasil. \\ ${ }^{2}$ Universidade Federal de Alagoas - UFAL, Arapiraca, Alagoas, Brasil. \\ ${ }^{3}$ Universidade Federal do Vale do São Francisco - UNIVASF, Petrolina, Pernambuco, Brasil. \\ ${ }^{4}$ Instituto de Medicina Integral Prof. Fernando Figueira - IMIP/UPAE, Petrolina, Pernambuco, Brasil.
}

\section{Resumo}

Introdução: A hanseníase é uma doença tropical negligenciada causada por Mycobacterium leprae. O Brasil é um dos principais países endêmicos para a doença, persistindo como problema de saúde pública. Além disso, é a principal doença infecciosa causadora de incapacidades físicas. Objetivos: Analisar a magnitude e os fatores associados à limitação de atividade nos casos novos de hanseníase diagnosticados no centro de referência do nordeste do Brasil. Métodos: Trata-se de estudo transversal envolvendo 50 pessoas com diagnóstico de hanseníase. Foram coletadas variáveis clínicas e sociodemográficas além de aplicação da escala SALSA para análise da limitação funcional. Empregou-se regressão logística com cálculo de odds ratio. Resultados: A presença de limitações funcionais foi registrada em $32 \%(n=16)$ dos casos analisados, destacando-se sexo feminino $(56,3 \%)$, idosos $(37,5 \%)$, baixa escolaridade $(87,6 \%)$, forma dimorfa (62,5\%), classificação multibacilar $(75,0 \%)$ e grau 2 de incapacidade física $(50,0 \%)$. A limitação funcional esteve associada a faixa etária $\geq 45$ anos (OR 3,80; $p=0,047$ ), classificação multibacilar (OR 4,28; $p=0,021$ ) e escore OMP $\geq 6$ (OR 4,69; $p=0,041)$. Conclusão: Observou-se elevada frequência de pessoas com incapacidade físicas. Os fatores associados à limitação funcional foram idade igual ou superior a 45 anos, classificação multibacilar e escore OMP maior ou igual a seis.

Palavras-chave: Hanseníase, Assistência Odontológica para Pessoas com Deficiências, Aplicações da Epidemiologia, Estudos Transversais, Mycobacterium leprae.
Como citar: Matos TS, Souza CDF, Moura JC, Fernandes TRMO, Mariano RS. Fatores associados à limitação de atividade em casos novos de hanseníase em município hiperendêmico do Nordeste, Brasil: estudo transversal. Rev Bras Med Fam Comunidade. 2021;16(43):2379. https://doi.org/10.5712/rbmfc16(43)2379

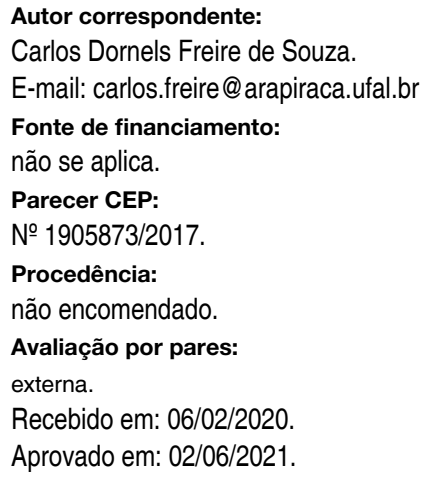




\begin{abstract}
Introduction: Leprosy is a neglected tropical disease caused by Mycobacterium leprae. Brazil is one of the main endemic countries for the disease, persisting as a public health problem. In addition, it is the main infectious disease that causes physical disabilities. Objectives: To analyze the magnitude and factors associated with activity limitation in new cases of leprosy diagnosed in the reference center in Northeastern Brazil. Methods: This is a cross-sectional study involving 50 people diagnosed with leprosy. Clinical and sociodemographic variables were collected in addition to the application of the SALSA scale for the analysis of functional limitations. Logistic regression was used with the calculation of odds ratio. Results: The presence of functional limitations was registered in $32 \%(n=16)$ of the cases analyzed, especially female $(56.3 \%)$, elderly $(37.5 \%)$, low education (87.6\%), dimorphic form (62.5\%), multibacillary classification (75.0\%), and degree 2 of physical disability (50.0\%). Functional limitation was associated with an age range $\geq 45$ years (OR $3.80 ; p=0.047$ ), multibacillary classification $(\mathrm{OR} 4.28 ; p=0.021)$, and OMP score $\geq 6$ (OR 4.69; $p=0.041$ ). Conclusion: There was a high frequency of people with physical disabilities. The factors associated with functional limitation were age equal to or greater than 45 years, multibacillary classification and OMP score greater than or equal to six.
\end{abstract}

Keywords: Leprosy, Dental Care for Disabled, Uses of Epidemiology, Cross-Sectional Studies, Mycobacterium leprae.

\title{
Resumen
}

Introducción: La lepra es una enfermedad tropical desatendida causada por Mycobacterium leprae. Brasil es uno de los principales países endémicos de la enfermedad, persistiendo como problema de salud pública. Además, es la principal enfermedad infecciosa que causa discapacidades físicas. Objetivo: Analizar la magnitud y los factores asociados a la limitación de la actividad en los nuevos casos de lepra diagnosticados en el centro de referencia del noreste de Brasil. Métodos: Se trata de un estudio transversal en el que participaron 50 personas diagnosticadas con lepra. Se recogieron variables clínicas y sociodemográficas además de la aplicación de la escala SALSA para el análisis de limitaciones funcionales. Se utilizó regresión logística con el cálculo del odds ratio. Resultados: La presencia de limitaciones funcionales se registró en el $32 \%(n=16)$ de los casos analizados, especialmente mujeres $(56,3 \%)$, ancianos $(37,5 \%)$, baja escolaridad $(87,6 \%)$, forma dismórfica $(62,5 \%)$, clasificación multibacilar $(75,0 \%)$ y grado 2 de discapacidad física (50,0\%). La limitación funcional se asoció con un rango de edad $\geq 45$ años (OR 3,$80 ; p=0,047$ ), clasificación multibacilar (OR 4,28; $p=0,021)$ y puntuación OMP $\geq 6(O R 4,69 ; p=0,041)$. Conclusión: Hubo una alta frecuencia de personas con discapacidad física. Los factores asociados a la limitación funcional fueron edad igual o superior a 45 años, clasificación multibacilar y puntuación OMP mayor o igual a seis.

Palabras clave: Lepra, Atención Dental para Personas con Discapacidades, Usos de la Epidemiología, Estudios Transversales, Mycobacterium leprae.

\section{INTRODUÇÃO}

A hanseníase é uma doença dermatoneurológica, crônica granulomatosa e infectocontagiosa causada por Mycobacterium leprae (M. leprae), que se manifesta, principalmente, por lesões cutâneas com diminuição de sensibilidade térmica, dolorosa e tátil e alterações em nervos periféricos, com elevado potencial incapacitante..$^{1-3}$

A Organização Mundial da Saúde (OMS) vem definindo ações estratégicas para o enfrentamento e a eliminação da hanseníase como problema de saúde pública. Em 2005, a "Estratégia global para 2006 2010" fundamentada na oferta de tratamento com poliquimioterapia (PQT) e na detecção precoce de casos. ${ }^{4}$ Em 2010, a "Estratégia global aprimorada para redução adicional da carga da hanseníase 2011-2015". Em 2011, o Ministério da Saúde lançou o "Plano integrado de ações estratégicas para a eliminação da hanseníase, filariose, esquistossomose e oncocercoce como problema de saúde pública, tracoma como causa de cegueira e controle das geo-helmintíases", que representou um avanço importante nas políticas públicas. Em 2016, a "Estratégia mundial de eliminação da hanseníase 2016-2020: acelerar a ação para um mundo sem lepra" foi lançada com objetivo de estimular a eliminação da doença em países prioritários. ${ }^{4-6}$

Mesmo com todos os esforços empreendidos, o Brasil ainda ocupa a primeira posição no mundo em coeficiente de prevalência, com uma taxa de prevalência de 1,50 por 10 mil habitantes em 2019; e o segundo em números absolutos de casos, ficando atrás apenas para Índia. Além disso, o Brasil é o único a não conseguir alcançar a meta de eliminação da doença como problema de saúde pública, ou seja, alcançar menos de 1 caso por 10.000 habitantes. ${ }^{7-9}$ 
Em 2019, foram identificados 202.185 novos casos de hanseníase em todo o mundo, o que corresponde a uma taxa de detecção de novos casos de 25,9 por milhão de habitantes. No Brasil, foram diagnosticados 27.864 casos novos de hanseníase nesse mesmo ano. Desses casos, 9,9\% ( $n=2.351)$ já apresentavam grau 2 de incapacidade física (GIF 2) no momento do diagnóstico. ${ }^{7,9}$

As incapacidades físicas, quando presentes, provocam limitações funcionais e de vida diária, contribuem para o estigma e geram comprometimento na participação social. ${ }^{8,10-13} \mathrm{Em}$ razão desse contexto, recomenda-se que os indivíduos diagnosticados com hanseníase sejam submetidos à avaliação neurológica simplificada e das limitações de atividade de vida diária, com a utilização da escala SALSA (screening of activity limitation and safety awareness). 2,8,14,15-17

O reconhecimento do perfil e dos fatores associados à limitação de atividades em decorrência da hanseníase podem contribuir para o desenvolvimento de ações de saúde no âmbito municipal que envolva os diferentes níveis de atenção, com destaque para a atenção primária à saúde (APS), que juntamente com a estratégia saúde da família cumpre um papel importante na promoção, prevenção e controle de agravos na saúde pública, sendo por tanto principal porta de entrada da população aos serviços de saúde. ${ }^{18-20}$

Na APS são realizadas ações de educação em saúde, que possibilitam prevenção, detecção precoce de casos e adesão ao tratamento da hanseníase, ainda é realizado a notificação de casos detectados, busca ativa de casos através da realização de exame de manchas, busca e avaliação dos contatos intradomiciliares, encaminhamento para atendimento especializado e acompanhamento do paciente na administração da dose supervisionada. ${ }^{18,20-22}$

O centro de referência em hanseníase, juntamente com a APS, desenvolve um trabalho em rede, voltado para melhor assistência e qualidade dos serviços prestados aos pacientes. Com base no exposto, este trabalho teve como objetivo analisar os fatores associados à limitação de atividade nos casos novos de hanseníase diagnosticados no centro de referência em hanseníase Dr. Altino Lemos Santiago, Juazeiro, Bahia, Brasil, 2018.

\section{MÉTODOS}

\section{Desenho de estudo, local e período}

Trata-se de um estudo transversal realizado no centro de referência em hanseníase Dr. Altino Lemos Santiago, situado no município de Juazeiro, Bahia, no período entre janeiro e junho de 2018. O município de estudo está situado na região norte do estado da Bahia, possui uma população estimada de 215 mil habitantes em 2018 (Figura 1), ${ }^{23}$ sendo considerado hiperendêmico para a hanseníase desde 2013 (coeficiente de detecção $\geq 40$ casos novos/100 mil habitantes). ${ }^{24} \mathrm{Em} 2018$, o município de Juazeiro registrou 171 casos novos da doença na população geral (79,46/100 mil habitantes) e 9 casos em menores de 15 anos (16,69/100 mil habitantes) e 11 pessoas com GIF 2 no diagnóstico, (5,1/um milhão de habitantes). ${ }^{25}$

Juazeiro é considerada referência no manejo da hanseníase na região norte da Bahia. A rede de atenção primária à saúde (APS), em 2018, era composta por 60 equipes de saúde da família e 93,34\% de cobertura. ${ }^{26}$ Mesmo assim, no âmbito intramunicipal, o diagnóstico da doença tem sido centralizado na unidade de referência. A APS tem papel destacado no acompanhamento do tratamento da doença, mas tímida participação no diagnóstico. O programa de referência em hanseníase conta com dois médicos e um fisioterapeuta, além de visitadoras sanitárias e duas técnicas de enfermagem. 




Figura 1. Mapa de localização de Juazeiro-Bahia. Fonte: Elaboração própria.

O fluxo de atendimento na rede municipal se dá da seguinte forma: diante de caso suspeito na unidade de saúde da família, o médico pode realizar o diagnóstico e iniciar o tratamento. Havendo dúvida, o paciente é referenciado para o centro Dr. Altino Lemos Santiago. Uma vez confirmado, é contrarreferenciado para a unidade de saúde realizar a notificação e o acompanhamento mensal. Em menores de 15 anos, o diagnóstico somente é feito do centro de referência. Como foi destacado, o diagnóstico está centralizado no centro de referência.

\section{População do estudo}

Foram convidados todos os casos novos de hanseníase diagnosticados entre janeiro e junho de 2018 no centro de referência municipal. Nesse período, foram diagnosticados 58 casos novos em residentes em Juazeiro. Esses indivíduos foram convidados a participar do estudo, havendo recusa de oito pessoas. Salienta-se que a população não participou do processo de planejamento do estudo. No entanto, uma cartilha final com os resultados do estudo foi produzida e encaminhada aos pacientes e um relatório encaminhado ao gestor do programa municipal de hanseníase.

\section{Variáveis analisadas}

Foram incluídas as seguintes variáveis: sexo (masculino e feminino), faixa etária (0-14, 15-29, 30-44, 45-59, 60 ou mais), escolaridade (analfabeto, ensino fundamental incompleto, ensino fundamental completo, ensino médio incompleto, ensino médio completo, ensino superior incompleto e ensino superior completo), forma clínica (indeterminada, tuberculoide, dimorfa e virchowiana), classificação operacional (paucibacilar e multibacilar), grau de incapacidade física-GIF (zero, um, dois), escore olhos-mãos-pés (OMP) e tempo (em meses) até o diagnóstico. 
O grau de incapacidade foi determinado a partir da avaliação neurológica dos olhos, das mãos e dos pés, o seu resultado é expresso em valores que variam de zero a dois, sendo zero se não houver comprometimento neural; um para diminuição ou perda da sensibilidade e dois para presença de incapacidades e deformidades. O escore olho-mão-pé ( $E H F$ - eye-hand-foot) é determinado a partir da soma do grau máximo de incapacidade para cada um dos seis locais do corpo (olhos direito e esquerdo, mãos direita e esquerda e pés direito e esquerdo), podendo variar de 0 a $12 .{ }^{14,27,28}$

O desfecho limitação de atividade se deu por meio da escala SALSA (screening of activity limitation and safety awareness), versão brasileira e validada, que tem suporte na classificação internacional de funcionalidade (CIF), é formada por 20 questões padronizadas capazes de avaliar domínios relacionados a acuidade visual, mobilidade dos pés, autocuidado, trabalho e destreza das mãos, gerando os escores de triagem de limitação de atividade e de consciência de risco de pessoas acometidas por neuropatias


(limitação leve); 40-49 (limitação moderada); 50-59 (limitação severa); $60-80$ (limitação muito severa). ${ }^{16,17,28}$

\section{Procedimentos de coleta de dados}

Inicialmente, os indivíduos obtiveram o diagnóstico da hanseníase realizado pelo profissional dermatologista do serviço. Em seguida, foram convidados a participar do estudo. Nesse momento, foram explicados todos os objetivos, procedimentos e importância da pesquisa. O aceite em participar da pesquisa foi oficializado por meio da assinatura do termo de consentimento livre e esclarecido (TCLE).

A avaliação das funções neurais e do grau de incapacidade física (GIF) deve ser realizada em três momentos nos pacientes hansenianos, no diagnóstico, durante o tratamento e após a alta. Na admissão a avaliação é realizada a fim de estimar o risco de desenvolvimento de sequelas neurofuncionais, e verificar a presença de incapacidade já instaladas no diagnóstico, fator indicativo que o diagnóstico ocorreu tardiamente. Estudos apontam que as formas multibacilares representam maiores chances de desenvolvimento de incapacidades. , $^{8,29,30}$

A coleta dos dados se deu no momento do diagnóstico, a partir da aplicação da escala SALSA (versão 1.0, em português), da avaliação neurológica simplificada e do preenchimento da ficha de dados sociodemográficos. Esses procedimentos foram realizados por um profissional fisioterapeuta devidamente treinado e em sala destinada a esta finalidade.

Os oito casos que recusaram participar da pesquisa também foram avaliados, todavia, suas avaliações não foram incluídas no estudo. Todos os pacientes que apresentaram alguma alteração nas avaliações foram encaminhados para acompanhamento da equipe no próprio centro de referência (médico e fisioterapeuta) ou outros serviços credenciados e/ou especialistas, quando necessário. Esta é a rotina do serviço e recomendação do Ministério da Saúde.

\section{Análise estatística}

Após a coleta, dos dados foram digitados em planilha eletrônica do Microsoft Excel e analisados utilizando o software IBM SPSS Statistics for Windows, Version 22.0 (IBM Corp. Released 2013. Armonk, NY: IBM Corp.). Foram utilizados como testes estatísticos: Kolmogorov-Smirnov para avaliação da normalidade 
dos dados, teste U-Mann-Whitney para comparação dos escores de limitação e regressão logística com cálculo da odds ratio (OR) para identificação dos fatores associados à limitação funcional. Nessa última etapa, algumas variáveis policotômicas foram dicotomizadas: faixa etária ( $<45$ e $\geq 45$ anos) escore OMP $(<6$ e $\geq 6$ ) e escolaridade ( $\leq 7$ anos de estudo e $>7$ anos de estudo), tempo até o diagnóstico ( $\geq 6$ meses e $<6$ meses). Adotou-se intervalo de confiança de 95\% e nível de significância de 5\%.

\section{Aspectos éticos}

O estudo foi aprovado pelo comitê de ética em pesquisa (CEP) da Universidade Federal do Vale do São Francisco (UNIVASF) sob o parecer de número 1905873/2017. Não será possível compartilhar dados, mesmo anonimizados, por questões operacionais.

\section{RESULTADOS}

Dos 50 casos novos de hanseníase incluídos no estudo, observou-se igual distribuição entre os sexos. A presença de limitações funcionais foi registrada em $32 \%(n=16)$ dos indivíduos, destacando-se a população feminina $(56,3 \%)$, idosos $(37,5 \%)$, baixa escolaridade - até o ensino fundamental $(87,4 \%)$, forma clínica dimorfa $(62,5 \%)$, classificação operacional multibacilar $(75,0 \%)$ e grau 2 de incapacidade física $(50,0 \%)$ (Tabela 1$)$.

O escore OMP foi maior nos indivíduos com limitações funcionais ( $p=0,010$ e $p=0,001$, respectivamente) (Tabela 2).

A regressão logística mostrou associação entre a presença de limitação funcional e as variáveis faixa etária $\geq 45$ anos (OR 3,$80 ; p=0,047$ ), formas multibacilares (OR 4,28; $p=0,021$ ) e escore OMP $\geq 6$ (OR 4,69; $p=0,041)$. Destaca-se que $75 \%(n=12)$ dos indivíduos com limitação possuíam idade $\geq 45$ anos e apresentavam classificação operacional multibacilar (Tabela 3).

\section{DISCUSSÃO}

Juazeiro na Bahia, cidade considerada hiperendêmica para hanseníase, é uma das 40 cidades, contempladas pela portaria $n^{\circ} 2.556$, de 28 de outubro de 2011, que define critérios de seleção para municípios prioritários para ações de controle da hanseníase. ${ }^{31}$ Tem apresentado bons indicadores epidemiológicos no que se refere à qualidade dos serviços de enfrentamento à hanseníase, fato justificado pela elevada cobertura da atenção primária à saúde no município e de centro de referência especializado. ${ }^{21}$

O aumento na cobertura da atenção primária e a capacitação das equipes de saúde são estratégias adotadas pelo Ministério da Saúde para o controle da doença em municípios endêmicos. Estando situada próximo à vida das pessoas, onde elas vivem e trabalham, as unidades de saúde possibilitam o diagnóstico precoce, o maior acesso ao tratamento, a prevenção de incapacidades, à medida que os pacientes são acompanhados pelas unidades de saúde, reduz-se o risco de abandono e aumenta a proporção de cura dos casos. ${ }^{20,22}$ Nesse sentido, os resultados deste trabalho devem fomentar o desenvolvimento de estratégias que ampliação da capacidade da APS no diagnóstico e manejo da hanseníase.

Apesar do conhecimento em relação às deficiências e incapacidades físicas associadas à doença, e do entendimento de que o paciente necessita de atendimento integral, há de ressaltar que a aplicação da 
Tabela 1. Caracterização sociodemográfica e epidemiológica dos casos novos de hanseníase, segundo presença/ausência de limitação funcional, diagnosticados no centro de referência Dr. Altino Lemos Santiago. Juazeiro, Bahia, 2018.

\begin{tabular}{|c|c|c|c|c|}
\hline \multirow[t]{2}{*}{ Variável } & \multicolumn{2}{|c|}{$\begin{array}{c}\text { Sem limitação } \\
34(68 \%)\end{array}$} & \multicolumn{2}{|c|}{$\begin{array}{l}\text { Com limitação } \\
16(32 \%)\end{array}$} \\
\hline & $\mathbf{n}$ & $\%$ & $\mathbf{n}$ & $\%$ \\
\hline \multicolumn{5}{|l|}{ Sexo } \\
\hline Masculino & 18 & $(52,9)$ & 7 & $(43,7)$ \\
\hline Feminino & 16 & $(47,1)$ & 9 & $(56,3)$ \\
\hline \multicolumn{5}{|l|}{ Faixa etária } \\
\hline $0-14$ & 1 & $(2,9)$ & 1 & $(6,2)$ \\
\hline $15-29$ & 5 & $(14,7)$ & 0 & $(0,0)$ \\
\hline $30-44$ & 14 & $(41,2)$ & 4 & $(25,0)$ \\
\hline $45-59$ & 10 & $(29,4)$ & 5 & $(31,3)$ \\
\hline 60 ou mais & 4 & $(11,8)$ & 6 & $(37,5)$ \\
\hline \multicolumn{5}{|l|}{ Escolaridade } \\
\hline Analfabeto & 2 & $(5,8)$ & 3 & $(18,7)$ \\
\hline Ensino fundamental incompleto & 16 & $(47,1)$ & 10 & $(62,4)$ \\
\hline Ensino fundamental completo & 4 & $(11,8)$ & 1 & $(6,3)$ \\
\hline Ensino médio incompleto & 2 & $(5,9)$ & 1 & $(6,3)$ \\
\hline Ensino médio completo & 7 & $(20,6)$ & 0 & $(0,0)$ \\
\hline Ensino superior incompleto & 1 & $(2,9)$ & 1 & $(6,3)$ \\
\hline Ensino superior completo & 2 & $(5,9)$ & 0 & $(0,0)$ \\
\hline \multicolumn{5}{|l|}{ Forma clínica } \\
\hline Indeterminada & 4 & 11,8 & 2 & 12,5 \\
\hline Tuberculoide & 16 & 47,0 & 2 & 12,5 \\
\hline Dimorfa & 11 & 32,4 & 10 & 62,5 \\
\hline Virchowiana & 3 & 8,8 & 2 & 12,5 \\
\hline \multicolumn{5}{|l|}{ Classificação operacional } \\
\hline Paucibacilar & 20 & 58,8 & 4 & 25,0 \\
\hline Multibacilar & 14 & 41,2 & 12 & 75,0 \\
\hline \multicolumn{5}{|l|}{ Grau de incapacidade física } \\
\hline Grau 0 & 9 & 26,5 & 2 & 12,5 \\
\hline Grau 1 & 18 & 52,9 & 6 & 37,5 \\
\hline Grau 2 & 7 & 20,6 & 8 & 50,0 \\
\hline
\end{tabular}

Tabela 2. Comparação dos escores OMP e tempo até o diagnóstico, segundo presença/ausência de limitação funcional dos casos novos de hanseníase diagnosticados no centro de referência Dr. Altino Lemos Santiago. Juazeiro, Bahia, 2018.

\begin{tabular}{llll}
\hline Escalas quantitativas $^{1}$ & Sem limitação & Com limitação & $\boldsymbol{p}$-valor \\
\hline Escore OMP (Mediana e IIQ) & $2,0(3)$ & $3,5(4)$ & 0,010 \\
Tempo até o diagnóstico - em meses (Mediana e IIQ) & $8,5(14)$ & $9,5(19)$ & 0,707 \\
\hline
\end{tabular}

Legenda: 1Teste U-Mann-Whitney; IIQ: Intervalo interquartil. 
Tabela 3. Fatores associados à presença de limitação de atividade no diagnóstico dentre os casos de hanseníase diagnosticados no Centro de Referência Dr. Altino Lemos Santiago. Juazeiro, Bahia, 2018.

\begin{tabular}{|c|c|c|c|c|c|c|}
\hline \multirow{2}{*}{ Variável } & \multirow{2}{*}{$\begin{array}{l}\text { Total } \\
\text { n (\%) }\end{array}$} & \multicolumn{2}{|c|}{ Limitação funcional } & \multirow{2}{*}{ p-valor } & \multirow{2}{*}{ OR1 } & \multirow{2}{*}{ IC 95\% } \\
\hline & & Sim & Não & & & \\
\hline \multicolumn{7}{|l|}{ Sexo } \\
\hline Masculino & $25(50,0)$ & $7(43,8)$ & $18(52,9)$ & \multirow{2}{*}{0,541} & \multirow{2}{*}{1,44} & \multirow{2}{*}{$0,44-4,78$} \\
\hline Feminino & $25(50,0)$ & $9(56,2)$ & $16(47,1)$ & & & \\
\hline \multicolumn{7}{|l|}{ Faixa etária* } \\
\hline$<45$ & $23(46,0)$ & $4(25,0)$ & $19(55,9)$ & \multirow{2}{*}{0,047} & \multirow{2}{*}{3,80} & \multirow{2}{*}{$1,01-14,21$} \\
\hline$\geq 45$ & $27(54,0)$ & $12(75,0)$ & $15(44,1)$ & & & \\
\hline \multicolumn{7}{|l|}{ Escolaridade } \\
\hline$\leq 7$ anos de estudo & $31(62,0)$ & $13(81,2)$ & $18(52,9)$ & \multirow{2}{*}{0,060} & \multirow{2}{*}{3,85} & \multirow{2}{*}{$0,92-16,01$} \\
\hline$>$ de 7 anos de estudo & $19(38,0)$ & $3(18,8)$ & $16(47,1)$ & & & \\
\hline \multicolumn{7}{|c|}{ Classificação operacional ${ }^{\star}$} \\
\hline Multibacilar & $26(52,0)$ & $12(75,0)$ & $14(41,2)$ & \multirow{2}{*}{0,021} & \multirow{2}{*}{4,28} & \multirow{2}{*}{$1,14-16,07$} \\
\hline Paucibacilar & $24(48,0)$ & $4(25,0)$ & $20(58,8)$ & & & \\
\hline \multicolumn{7}{|l|}{ Escore OMP* } \\
\hline$<6$ & $42(84,0)$ & $11(68,7)$ & $31(91,2)$ & \multirow{2}{*}{0,041} & \multirow{2}{*}{4,69} & \multirow{2}{*}{$1,99-22,94$} \\
\hline$\geq 6$ & $8(16,0)$ & $5(31,3)$ & $3(8,8)$ & & & \\
\hline \multicolumn{7}{|l|}{ Incapacidade física } \\
\hline Sim & $39(78,0)$ & $14(87,5)$ & $25(73,5)$ & \multirow{2}{*}{0,277} & \multirow{2}{*}{2,52} & \multirow{2}{*}{$0,47-13,3$} \\
\hline Não & $11(22,0)$ & $2(12,5)$ & $9(26,5)$ & & & \\
\hline \multicolumn{7}{|l|}{ Tempo até o diagnóstico } \\
\hline$\geq 6$ meses & $18(36,0)$ & $5(31,3)$ & $13(38,2)$ & \multirow{2}{*}{0,633} & \multirow{2}{*}{1,36} & 020101 \\
\hline$<6$ meses & $32(64,0)$ & $11(68,7)$ & $21(61,8)$ & & & $0,38-4,8 \mid$ \\
\hline História de hanseníase $n$ & & & & & & \\
\hline Sim & $22(44,0)$ & $7(43,7)$ & $15(44,1)$ & 0000 & 101 & חמת חמת \\
\hline Não & $28(56,0)$ & $9(56,3)$ & $19(55,9)$ & 0,500 & (1, I & $0,30-3,30$ \\
\hline
\end{tabular}

escala SALSA é recomendação para a atenção aos pacientes com hanseníase, não sendo um protocolo essencial. No município estudado, essa escala não é utilizada na rotina assistencial, deixando lacunas sobre como as incapacidades afetam a realização das atividades da vida diária e as limitações funcionais de uma pessoa com hanseníase. ${ }^{10,14}$

Neste estudo, foi observada igual distribuição entre os sexos, predomínio de faixa etária economicamente ativa, baixa escolaridade, presença de incapacidades físicas em mais de dois terços da população estudada e limitação funcional em $32 \%$ dos indivíduos, estando associada à idade, classificação operacional e escore OMP. Resultados semelhantes foram observados em Bauru/SP, em investigação envolvendo 100 pacientes com hanseníase, no qual houve predomínio de casos multibacilares $(84,0 \%)$ e baixa escolaridade (53,0\%). ${ }^{32}$ Em investigação no estado do Amazonas, $81,0 \%$ dos casos eram multibacilares, $50,0 \%$ possuíam apenas o ensino primário e $26,2 \%$ tinham idade entre $31-40$ anos $^{2}$. Esse cenário reflete a necessidade de ampliação das medidas de enfrentamento já existentes.

Em estudo de série histórica, realizada no Nordeste entre 2001 e 2014, identificou-se tendência de aumento significativo (AAPC=4,0; IC95\%: 1,3-6,8) do coeficiente de detecção com grau 2 de incapacidade física, assim como nos casos multibacilares (AAPC=2,2; IC95\%: 0,1-4,3). ${ }^{33}$ Esses achados provavelmente 
estão associados ao negligenciamento da doença, ao longo período de incubação do bacilo, ao diagnóstico tardio e à provável endemia oculta pelo elevado percentual de casos multibacilares e casos com incapacidades visíveis, além do acometimento em maior proporção de pessoas com idade mais avançada. ${ }^{34}$

O perfil dos pacientes observado em todo o território nacional expressa o caráter de doença negligenciada, fortemente associada à pobreza e com estreita relação com as condições de vida e trabalho. ${ }^{33,35,36}$ Recentemente, um elevado número de investigações tem buscado compreender a influência dos determinantes sociais na dinâmica de transmissão da hanseníase no Brasil. ${ }^{35-40}$

Estudos realizados no próprio estado da Bahia mostraram que a manutenção da transmissão pode estar associada a: densidade domiciliar, proporção de extremamente pobres, renda per capita, desigualdade de renda e nível de ocupação. ${ }^{41,42}$ Por outro lado, em áreas endêmicas, é possível que as melhorias sociais gerais resultem, pelo menos em um primeiro momento, em aumento do número de diagnósticos, já que diminui a prevalência oculta. ${ }^{42}$ Impactos benéficos das políticas de transferência de renda, a exemplo do programa bolsa família e da expansão da rede de saúde, têm sido destacados como importantes mecanismos capazes de aumentar a adesão à poliquimioterapia e cura da hanseníase em casos multibacilares, além de interromper a transmissão da doença. ${ }^{43-48}$

Esses determinantes sociais também influenciam na ocorrência de incapacidades físicas ${ }^{42}$ e limitações funcionais. ${ }^{28}$ No município de Juazeiro/BA, mais de dois terços dos indivíduos já possuíam algum grau de incapacidade (1 ou 2), e 32,0\% apresentaram limitação funcional. Salienta-se que a existência de indivíduos com grau 2 de incapacidade e, ao mesmo tempo, sem limitação física pode ser explicada pela capacidade desses indivíduos em realizar as tarefas previstas no questionário. Isso sugere que a incapacidade física observada pode transmitir uma limitação aparente e não necessariamente uma barreira à realização de atividades.

Os achados observados corroboram outros estudos, como o realizado em Vitória da Conquista, também no estado da Bahia, no qual $28,7 \%$ dos pacientes com grau dois de incapacidade já possuíam limitação de atividade. ${ }^{10}$ Por outro lado, em Caruaru, Pernambuco, o percentual de indivíduos com limitação foi superior ao observado em Juazeiro e Vitória da Conquista, alcançando 56,7\%, somando-se as limitações leves, moderadas e graves. ${ }^{11}$ Essa diferença observada sinaliza que o diagnóstico da hanseníase em Juazeiro pode ocorrer mais precocemente, tendo em vista o elevado percentual de indivíduos sem limitações e não haver nenhum caso com limitação grave na população estudada.

Dentre os fatores associados à limitação funcional, destaca-se inicialmente a idade. ${ }^{17}$ Dentre os que apresentavam limitação, 37,5\% eram idosos (60 anos ou mais) e indivíduos com 45 anos ou mais possuíam uma chance 3,8 vezes maior de ter limitações funcionais quando comparados aos mais jovens. O envelhecimento proporciona modificações graduais e inevitáveis no organismo humano, ${ }^{49}$ a exemplo da fragilização do sistema imunológico e neuromuscular, fatores que ampliam a patogenicidade de $M$. leprae e tornam essa população mais vulnerável ao adoecimento e às incapacidades físicas e limitações funcionais e sociais. ${ }^{32,50}$

Em investigação conduzida por Matos et al. (2019), ${ }^{50}$ realizado no município de Juazeiro, envolvendo 273 casos de hanseníase em idosos registrados no período 2010-2016, fatores como sexo masculino, pessoas com $\geq 75$ anos de idade e com classificação operacional multibacilar apresentaram maior risco de desenvolvimento de incapacidades físicas. 
Um segundo fator de risco refere-se à forma clínica da doença. Pacientes classificados com a forma dimorfa e vichowiana apresentaram chance 4,28 maior de desenvolverem limitações funcionais quando comparados aos indivíduos paucibacilares. Esses achados corroboram com a literatura brasileira. ${ }^{8,28,50,51}$ Em revisão sistemática de Paula et al. (2019), ${ }^{52}$ considerando 28 estudos e englobando 39.192 pacientes, a chance de indivíduos com formas clínicas multibacilares desenvolverem incapacidades físicas foi quatro vezes superior ao de indivíduos multibacilares (OR 4,32), muito semelhante ao observado neste estudo.

A forma dimorfa apresenta característica imunológica de instabilidade, ora estando no polo de imunidade celular mediada ora no polo humoral. Já na forma virchowiana predomina o polo humoral. 1,3,53,54 Este contexto imunológico associado à alta carga bacilar resulta em maior dano neural, leva à ocorrência de incapacidades físicas e limitações funcionais. ${ }^{2,51}$ Nesse sentido, os danos neurais elevam o escore OMP, de modo que indivíduos com escores maiores que seis possuíam uma chance 4,69 vazes maior de apresentarem limitações funcionais, sendo este o terceiro fator associado observado neste estudo. A relação direta entre os escores OMP e SALSA foi demonstrada em estudo envolvendo 69 pacientes no município de Sobral, Ceará. ${ }^{27}$

A escala de SALSA pode ser utilizada com a finalidade de triagem, comparar um indivíduo com um grupo, comparar um grupo entre si, comparar um indivíduo consigo mesmo em diferentes momentos do tratamento, estabelecer intervenções individuais, realizar planejamento de projetos terapêuticos, avaliar intervenções ou projetos e pesquisa, sendo importante a sua implementação em todos os níveis de atenção à saúde, para utilização em indivíduos acometidos por hanseníase, diabetes mellitus e neuropatias periféricas. ${ }^{14,16,17,28}$

Mesmo considerando os cuidados metodológicos, o estudo possui limitações. Advogamos que estudos maiores e multicêntricos podem ajudar a compreender melhor a magnitude e os fatores associados à limitação funcional em indivíduos com hanseníase. Em razão da reduzida, os intervalos de confiança foram amplos e frequentemente admitiram tanto redução, quanto aumento importante das chances de limitação funcional. Uma segunda limitação diz respeito ao próprio tipo de estudo (transversal), uma vez que não é possível avaliar o impacto do tratamento na limitação funcional e nem a evolução das incapacidades físicas, além da própria estrutura dos serviços de saúde.

\section{CONCLUSÃO}

O estudo mostrou $32 \%$ de prevalência de limitações funcionais em casos novos de hanseníase no município de Juazeiro, Bahia. No grupo de indivíduos com limitações funcionais, destacou-se a população feminina, idosos, baixa escolaridade, forma clínica dimorfa, classificação operacional multibacilar e grau 2 de incapacidade física. Os fatores associados à maior limitação foram a faixa etária $\geq 45$ anos, as formas clínicas multibacilares e o escore OMP.

Ações que possibilitem o diagnóstico precoce e o tratamento oportuno, bem como a avaliação da limitação funcional nos casos de hanseníase por meio da escala SALSA na rotina dos serviços de saúde podem contribuir para a prevenção da incapacidade física e das limitações funcionais causadas pela hanseníase. 


\section{Contribuição dos autores}

Matos TS: contribuiu na concepção e no delineamento do trabalho, na aquisição, análise e interpretação dos dados, na elaboração do rascunho do trabalho, na sua revisão crítica para conteúdo intelectual importante e na aprovação final da versão a ser publicada.

Fernandes TRMO: contribuiu delineamento do trabalho e na interpretação dos dados, na revisão crítica para conteúdo intelectual importante e aprovação final da versão a ser publicada.

Moura JC: contribuiu delineamento do trabalho e na interpretação dos dados, na revisão crítica para conteúdo intelectual importante e aprovação final da versão a ser publicada.

Mariano RS: contribuiu na concepção do trabalho, na interpretação dos dados, na revisão crítica para conteúdo intelectual importante e aprovação final da versão a ser publicada.

Souza CDF: contribuiu na concepção e no delineamento do trabalho, na aquisição, análise e interpretação dos dados, na elaboração do rascunho do trabalho, na sua revisão crítica para conteúdo intelectual importante e na aprovação final da versão a ser publicada.

Todos os autores concordam em prestar contas de todos os aspectos do trabalho, assegurando que as questões relacionadas à acurácia ou integridade de qualquer parte do trabalho sejam devidamente investigadas e resolvidas.

\section{Conflito de interesses}

Sem conflitos de interesses.

\section{REFERÊNCIAS}

1. Talhari C, Talhari S, Penna GO. Clinical aspects of leprosy. Clin Dermatol. 2015;33(1):26-37. DOI: https://doi.org/10.1016/j. clindermatol.2014.07.002 PMID: 25432808 DOI: https://doi.org/10.1016/j.clindermatol.2014.07.002

2. Aben-Athar CYUP, Lima SS, Ishak R, Vallinoto ACR. Assessment of the sensory and physical limitations imposed by leprosy in a Brazilian Amazon Population. Rev Soc Bras Med Trop. 2017 Mar/Abr;50(2):223-8. DOI: https://doi.org/10.1590/0037-8682-0493-2016 3.

Fischer M. Leprosy - an overview of clinical features, diagnosis, and treatment. J Ger Soc Dermatol. 2017 Ago;15(8):801-27. DOI: https:// doi.org/10.1111/ddg.13301

4. Organização Mundial da Saúde (OMS). Organização Pan-Americana de Saúde (OPAS). Estratégia global aprimorada para redução adicional da carga da hanseníase (período do plano: 2011-2015) [Internet]. Brasília (DF): OMS/OPAS; 2010 ; [acesso em 2021 Mar 18] . Disponível em: http://bvsms.saude.gov.br/bvs/publicacoes/estrategia_global_aprimorada_reducao_hanseniase.pdf

5. Ministério da Saúde (BR). Plano integrado de ações estratégicas de eliminação da hanseníase, filariose, esquistossomose e oncocercose como problema de saúde pública, tracoma como causa de cegueira e controle das geohelmintíases: plano de ação $2011-2015$ [Internet]. Brasília (DF): Ministério da Saúde, 2012; [acesso em 2021 Mar 20]. Disponível em: http://bvsms.saude.gov.br/bvs/publicacoes/plano_ integrado_acoes_estrategicas_hanseniase.pdf

6. Organização Mundial da Saúde (OMS). Estratégia mundial de eliminação da lepra 2016-2020: acelerar a ação para um mundo sem lepra [Internet]. Genebra: OMS; 2016; [acesso em 2021 Mar 18]. Disponível em: https://apps.who.int/iris/bitstream/hand le/10665/208824/9789290225201-pt.pdf

7. World Health Organization (WHO). Weekly epidemiological record. Relevé épidémiologique hebdomadaire [Internet]. 2020 Set; [citado 2021 Abr 11]; 36(95):417-40. Disponível em: http://www.who.int/wer

8. Souza CDF, Fernandes TRMO, Matos TS, Ribeiro Filho JM, Almeida GKA, Lima JCB, et al. Physical disability degree in the elderly population affected by leprosy in the state of Bahia, Brazil. Acta Fisiátrica. 2017 Jan;24(1):27-32. DOI: https://doi.org/10.5935/01047795.20170006 
9. Ministério da Saúde (BR). Boletim Epidemiológico Especial. Secretária de Vigilância em Saúde. Hanseníase - 2021 [Internet]. Brasília (DF): Ministério da Saúde; 2021; [acesso em 2021 Abr 06]; Disponível em: https://www.gov.br/saude/pt-br/media/pdf/2021/fevereiro/12/ boletim-hanseniase-_-25-01.pdf

10. Raposo MT, Reis MC, Caminha AVQ, Heukelbach J, Parker LA, Pastor-Valero M, et al. Grade 2 disabilities in leprosy patients from Brazil: need for follow-up after completion of multidrug therapy. PLoS Negl Trop Dis. 2018;12(7):e0006645. DOI: https://doi.org/10.1371/journal. pntd.0006645 PMID: 30011288

11. Brandão BP, Silva MCL, Andrade MCF, Silva LVC. Avaliação física e funcional de pacientes com hanseníase. Rev Enferm UFPE On line. 2015;9(Supl 8):9336-42. DOI: https://doi.org/10.5205/reuol.6812-75590-1-ED.0908sup201501

12. Adhikari B, Kaehler N, Chapman RS, Raut S, Roche P. Factors affecting perceived stigma in leprosy affected persons in Western Nepal. PLoS NegI Trop Dis. 2014 Jun;8(6):e2940. DOI: https://doi.org/10.1371/journal.pntd.0002940 PMID: 24901307

13. Morgado FFDR, Silveira EMKXD, Sales AM, Nascimento LPRD, Sarno EN, Nery JADC, et al. Cross-cultural adaptation of the EMIC stigma scale for people with leprosy in Brazil. Rev Saúde Pública. 2017;51(1):80. DOI: https://doi.org/10.11606/S1518-8787.2017051000167 PMID: 28876410

14. Ministério da Saúde (BR). Série A. Normas e Manuais técnicos. Cadernos de prevenção e reabilitação em hanseníase: normas e manuais técnicos. Manual de prevenção de incapacidades [Internet]. Brasília (DF): Ministério da Saúde; 2008. Disponível em: http://bvsms.saude. gov.br/bvs/publicacoes/manual_prevencao_incapacidades.pdf

15. Abdela SG, Van Henten S, Abegaz SH, Bayuh FB, Zewdu FT, Berhe FT, et al. Activity limitation and social participation restriction among leprosy patients in Boru Meda Hospital, Amhara Region, Ethiopia. PLoS Negl Trop Dis. 2020 Set;14(9):e0008702. DOI: https://doi. org/10.1371/journal.pntd.0008702

16. Ebenso, J, Fuzikawa P, Melchior H, Wexler R, Piefer A, Min CS, et al. Desenvolvimento de um pequeno questionário para rastreamento de limitação de atividades e conscientização sobre segurança (SALSA) em clientes afetados por hanseníase ou diabetes. Disabil Rehabil. 2007;29(9):689-700. DOI: https://doi.org/10.1080/09638280600926587

17. Nascimento DDS, Ramos Junior AN, Araújo OD, Macêdo SF, Silva GVD, Lopes WMPS, et al. Activity limitation and social participation restriction of people with leprosy: a cross-sectional analysis of magnitude and associated factors in a hyperendemic municipality in the state of Piaui, Brazil, 2001-2014. Epidemiol Serv Saude. 2020;29(3):e2019543.DOI: https://doi.org/10.5123/s1679-49742020000300012

18. Coêlho LS, Albuquerque KR, Maia NMFS, Carvalho LRB, Almeida CAPL, Silva MP.Vivência do enfermeiro da atenção básica nas ações de controle da hanseníase. Rev Enferm UFPE On line. 2015 Dez; [acesso em 2021 Abr 11]; 9(Supl 10):1411-7. Disponível em: https:// periodicos.ufpe.br/revistas/revistaenfermagem/article/download/10853/12072

19. Ministério da Saúde (BR). Portaria n².436, de 21 de setembro de 2017. Aprova a Política Nacional de Atenção Básica, estabelecendo a revisão de diretrizes para a organização da Atenção Básica, no âmbito do Sistema Único de Saúde (SUS) [Internet]. Diário Oficial da União, Brasília (DF), 22 set 2017; Seção 1:68; [acesso em 2021 Abr 07]. Disponível em: http://bvsms.saude.gov.br/bvs/saudelegis/ gm/2017/prt2436_22_09_2017.html

20. Gomes AKB, Azevedo SR, Galvão BS, Costa AC, Sousa FPG, Leite GA, et al. Plano de intervenção para melhorar o diagnóstico, tratamento e acompanhamento da hanseníase em uma estratégia saúde da família de Belém-PA. Rev Bras Pesq Saúde [Internet]. 2019 [citado 2021 Abr 09]; 2(4):3431-45. Disponível em: http://www.brazilianjournals.com/index.php/BJHR/article/view/2387 DOI: https://doi. org/10.34119/bjhrv2n4-105

21. Souza CDF, Matos TS. Análise de tendência dos indicadores de monitoramento e avaliação da qualidade dos serviços de hanseníase em município prioritário do Nordeste brasileiro. Rev Bras Pesq Saúde [Internet]. 2017 Out/Dez; [citado 2021 Abr 09]; 19(4):74-83. Disponível em: https://www.periodicos.ufes.br/rbps/article/download/19806/13228 DOI: https://doi.org/10.21722/rbps.v19i4.19806

22. Saltarelli RMF, Seixas DHT. Limites e possibilidades na atenção ao portador de hanseníase no âmbito da estratégia saúdem da família. Rev APS [Internet]. 2016 Out; [citado 2021 Abr 09]; 19(4):613-22. Disponível em: https://aps.ufjf.emnuvens.com.br/aps/article/view/2443/1041

23. Instituto Brasileiro de geografia e Estatística (IBGE). IBGE cidades 2019 [Internet]. Brasília (DF): IBGE; 2017 ; [acesso em 2021 Mar 16]. Disponível em: https://cidades.ibge.gov.br/brasil/ba/juazeiro

24. Ministério da Saúde (BR). Secretaria de Vigilância em Saúde. Departamento de Vigilância das Doenças Transmissíveis. Diretrizes para a vigilância, atenção e eliminação da hanseníase como problema de saúde pública: manual técnico-operacional [Internet]. Brasília (DF): Ministério da Saúde;2016; [acesso em 2021 Mar 28]. Disponível em: https://portalarquivos2.saude.gov.br/images/pdf/2016/fevereiro/04/ diretrizes-eliminacao-hanseniase-4fev16-web.pdf

25. Ministério da Saúde (BR). Portal da Saúde - SUS. Sistema de Informação de Notificação (SIN). Informações de Saúde (TABNET). Epidemiológicas e morbidade, hanseníase Juazeiro-Bahia, 2018 [Internet]. Brasília (DF): Ministério da Saúde; 2001 ; [acesso em 2021 Abr 12]. Disponível em: http://www2.datasus.gov.br/DATASUS/index.php?area=0203\&id=34622409\&VObj=http://tabnet.datasus.gov. $\mathrm{br} / \mathrm{cgi} /$ tabcgi.exe?sinannet/cnv/hansw 
26. Ministério da Saúde (BR). Secretaria de Atenção Primária à Saúde (SAPS). Departamento de Saúde da Família (DESF) Unidade Geográfica:NORDESTE - BA - JUAZEIRO. E-Gestor Atenção Básica. Cobertura da Atenção Básica. Período: janeiro de 2018 à dezembro de 2018 [Internet]. Brasília (DF): Ministério da Saúde; 2018; [acesso em 2021 Abr 11]. Disponível em: https://egestorab.saude.gov.br/ paginas/acessoPublico/relatorios/relHistoricoCoberturaAB.xhtml

27. Barbosa JC, Ramos Junior AN, Alencar MDJF, Castro CGJ. Pós-alta em hanseníase no Ceará: limitação da atividade funcional, consciência de risco e participação social. Rev Bras Enferm [Internet]. 2008 Nov; [citado 2021 Abr 02]; 61(spe):727-33. Disponível em: https://www.scielo.br/scielo.php?pid=S0034-71672008000700012\&script=sci_arttext\&tlng=pt DOI: https://doi.org/10.1590/S003471672008000700012

28. Monteiro LD, Alencar CH, Barbosa JC, Novaes CCBS, Silva RCP, Heukelbach J. Limited activity and social participation after hospital discharge from leprosy treatment in a hyperendemic area in north Brazil. Rev Bras Epidemiol [Internet]. 2014 Mar; [citado 2021 Abr 09]; 17(1):91-104. Disponível em: http://www.scielo.br/scielo.php?script=sci_arttext\&pid=S1415-790X2014000100091\&lng=en DOI: https:// doi.org/10.1590/1415-790X201400010008ENG

29. Monteiro MJDSD, Santos GM, Barreto MTS, Sousa SRV, Jesus RLR, Silva HJN. Perfil epidemiológico de casos de hanseníase em um estado do nordeste brasileiro. Rev Aten Saúde. 2017 Out/Dez;15(54):21-8. DOI: https://doi.org/10.13037/ras.vol15n54.4766

30. Ribeiro Júnior AF, Vieira MA, Caldeira AP. Perfil epidemiológico da hanseníase em uma cidade endêmica no Norte de Minas Gerais. Rev Bras Clin Med [Internet]. 2012 Ju/Ago; [citado 2020 Dez 20]; 10(4):272-7. Disponível em: http://files.bvs.br/upload/S/1679-1010/2012/ v10n4/a3046.pdf

31. Ministério da Saúde (BR). Portaria no 2.556, de 28 de outubro de 2011. Estabelece mecanismo de repasse financeiro do fundo nacional de saúde aos fundos de saúde estaduais, do distrito federal e municipais, por meio do piso variável de vigilância e promoção da saúde, para implantação, implementação e fortalecimento da vigilância epidemiológica de hanseníase, tracoma, esquistossomose e geohelmintíases. [Internet]. Diário Oficial da União, Brasília (DF), 28 out 2011; Seção 1: 1; [acesso em 2021 Abr 09]. Disponível em: http://bvsms.saude. gov.br/bvs/saudelegis/gm/2011/prt2556_28_10_2011.html

32. Calixto MF, Marciano LHC, Prado RBR, Nardi SMT, Marques T. Functioning and disability in leprosy. Indian J Lepr [Internet]. 2016 Fev; [citado 2021 Mar 18]; 88(3):137-46. Disponível em: http://www.ijl.org.in/2016/1\%20MF\%20Calixto\%20et\%20al\%20(137-146).pdf

33. Souza EA, Ferreira AF, Boigny RN, Alencar CH, Heukelbach J, Martins-Melo FR, et al. Leprosy and gender in Brazil: trends in an endemic area of the Northeast region, 2001-2014. Rev Saúde Pública. 2018;52:20. DOI: https://doi.org/10.11606/s1518-8787.2018052000335

34. Lima LNGC, Paz JLP, Silvestre MPSCA, Moura LS, Furlaneto IP, Lima KVB. BCG vaccination status, age, and gender as risk factors for leprosy in endemic areas in the Brazilian Amazon. Infect Dis Rep. 2020;12(3):97-104. DOI: https://doi.org/10.3390/idr12030019

35. Pescarini JM, Strina A, Nery JS, Skalinski LM, Andrade KVF, Penna MLF, et al. Socioeconomic risk markers of leprosy in high-burden countries: a systematic review and meta-analysis. PLoS Negl Trop Dis. 2018 Jul;12(7):e0006622. DOI: https://doi.org/10.1371/journal. pntd.0006622 PMID: 29985930

36. Nery JS, Ramond A, Pescarini JM, Alves A, Strina A, Ichihara MY, et al. Socioeconomic determinants of leprosy new case detection in the 100 million Brazilian cohort: a population-based linkage study. Lancet Glob Health. 2019 Set;7(9):e1226-e36. DOI: https://doi. org/10.1016/S2214-109X(19)30260-8

37. Silva CLM, Fonseca SC, Kawa H, Palmer DOQ. Spatial distribution of leprosy in Brazil: a literature review. Rev Soc Bras Med Trop. 2017 Jul/Ago;50(4):439-49. DOI: https://doi.org/10.1590/0037-8682-0170-2016

38. Ferreira AF, Amorim SE, Soledad MGG, Silva RA, Corona F, Silveira LM, et al. Leprosy in the North and Northeast regions of Brazil: an integrated spatiotemporal approach. Trop Med Int Health. 2020 Nov;25(2):193-208. DOI: https://doi.org/10.1111/tmi. 13343

39. Hacker MA, Sales AM, Duppre NC, Sarno EN, Moraes MO. Leprosy incidence and risk estimates in a 33-year contact cohort of leprosy patients. Sci Rep. 2021 Jan;11(1):1947. DOI: https://doi.org/10.1038/s41598-021-81643-4

40. Souza CDF, Santos VS, Nery JS, Fernandes TRMO, Magalhães MAFM. Can the municipal social deprivation index influence the time trend of the leprosy detection rate? Rev Soc Bras Med Trop. 2020;54:e20200228. DOI: https://doi.org/10.1590/0037-8682-0228-2020 PMID: 33206881

41. Amorim SE, Fuentes FA, Heukelbach J, Nzundu BR, Alencar CH, Júnior Novaes RA. Epidemiology and spatiotemporal patterns of leprosy detection in the state of Bahia, Brazilian Northeast Region, 2001-2014. Trop Med Infect Dis. 2018 Jul;3(3):79. DOI: https://doi. org/10.3390/tropicalmed3030079

42. Souza CDF, Luna CF, Magalhães MDAFM. Spatial modeling of leprosy in the state of Bahia and its social determinants: a study of health inequities. An Bras Dermatol. 2019 Mar/Apr;94(2):182-91. DOI: https://doi.org/10.1590/abd1806-4841.20197554 
43. Pescarini JM, Williamson E, Nery JS, Ramond A, Ichihara MY, Fiaccone RL, et al. Effect of a conditional cash transfer programme on leprosy treatment adherence and cure in patients from the nationwide 100 million Brazilian cohort: a quasi-experimental study. Lancet Infect Dis. 2020 Mai;20(5):618-27. DOI: https://doi.org/10.1016/S1473-3099(19)30624-3

44. Santos ÁN, Costa AKAN, Souza JÉR, Alves KAN, Oliveira KPMM, Pereira ZB. Epidemiological profile and tendency of leprosy in people younger than 15 years. Rev Esc Enferm USP. 2020;54:e03659. DOI:https://doi.org/10.1590/S1980-220X2019016803659 PMID: 33295532

45. Souza EA, Heukelbach J, Oliveira MLW, Ferreira AF, Sena Neto SA, Raposo MT, et al. Low performance of operational indicators for leprosy control in the state of Bahia: spatiotemporal patterns, 2001-2014. Rev Bras Epidemiol. 2020;23:e200019. DOI: https://doi. org/10.1590/1980-549720200019 PMID: 32159629

46. Ferreira AF, Sousa EA, García GSM, Reis AS, Corona F, Lima MS, et al. Leprosy in the North and Northeast regions of Brazil: an integrated spatiotemporal approach. Trop Med Int Health. 2020 Nov;25(2):193-208. DOI: https://doi.org/10.1111/tmi.13343

47. Nery JS, Pereira SM, Rasella D, Penna MLF, Aquino R, Rodrigues LC, et al. Effect of the Brazilian conditional cash transfer and primary health care programs on the new case detection rate of leprosy. PLoS Neglect Trop Dis.2014 Nov;8(11):e3357.DOI: https://doi.org/10.1371/ journal.pntd.0003357

48. Andrade KVF, Nery JS, Oliveira PG, Penna L, Barreto M, Pereira SM. Effect of Brazil's conditional cash transfer programme on the new case detection rate of leprosy in children under 15 years old. Lepr Rev [Internet].2018; [citado 2021 Mar 18]; 89:13-24. Disponível em: https://www. researchgate.net/profile/Kaio_Vinicius_Freitas_De_Andrade/publication/324342373_Effect_of_Brazil's_Conditional_Cash_Transfer_ Programme_on_the_new_case_detection_rate_of_leprosy_in_children_under_15_years_old/links/5acb66f80f7e9bcd5199927d/ Effect-of-Brazils-Conditional-Cash-Transfer-Programme-on-the-new-case-detection-rate-of-leprosy-in-children-under-15-years-old.pdf DOI: https://doi.org/10.47276/lr.89.1.13

49. Organização Mundial da Saúde (OMS). Relatório mundial de envelhecimento e saúde [Internet]. Geneva: OMS; 2015; [acesso em 2021 Abr 12]. Disponível em: https://apps.who.int/iris/bitstream/handle/10665/186468/WHO_FWC_ALC_15.01_por.pdf;js

50. Matos TS, Carmo RFD, Santos FGB, Souza CDF. Leprosy in the elderly population and the occurrence of physical disabilities: Is there cause for concern? An Bras Dermatol. 2019 Mar/Abr;94(2):243-5. DOI: https://doi.org/10.1590/abd1806-4841.20198067

51. Monteiro LD, Alencar CHMD, Barbosa JC, Braga KP, Castro MDD, Heukelbach J. Incapacidades físicas em pessoas acometidas pela hanseníase no período pós-alta da poliquimioterapia em um município no Norte do Brasil. Cad Saúde Pública. 2013;29(5):909-20. DOI: https://doi.org/10.1590/s0102-311x2013000500009

52. Paula HL, Souza CD, Silva SR, Martins-Filho PR, Barreto JG, Gurgel RQ, et al. Risk factors for physical disability in patients with leprosy: a systematic review and meta-analysis. JAMA Dermatol.2019 Ago;155(10):1120-8. DOI: https://doi.org/10.1001/jamadermatol.2019.1768

53. Saunderson P.The epidemiology of reactions and nerve damage. Lepr Rev.2000;71(Suppl 1):S106-S10. DOI: https://doi.org/10.5935/03057518.20000079 PMID: 11201864

54. Sales AM, Leon AP, Düppre NC, Hacker MA, Nery JAC, Sarno EN, et al. Leprosy among patient contacts: a multilevel study of risk factors. PLoS NegI Trop Dis. 2011 Mar;5(3):e1013. DOI: https://doi.org/10.1371/journal.pntd.0001013 